\title{
Formal Theory of Fun and Creativity
}

\author{
Jürgen Schmidhuber \\ IDSIA \& USI \& SUPSI \\ Switzerland \\ juergen@idsia.ch
}

To build a creative agent that never stops generating non-trivial \& novel \& surprising data, we need two learning modules: (1) an adaptive predictor or compressor or model of the growing data history as the agent is interacting with its environment, and (2) a general reinforcement learner. The LEARNING PROGRESS of (1) is the FUN or intrinsic reward of (2). That is, (2) is motivated to invent interesting things that (1) does not yet know but can easily learn. To maximize expected reward, in the absence of external reward (2) will create more and more complex behaviors that yield temporarily surprising (but eventually boring) patterns that make (1) quickly improve. We discuss how this principle explains science \& art \& music \& humor, and how to scale up previous toy implementations of the theory since 1991, using recent powerful methods for (1) prediction and (2) reinforcement learning. 